\title{
Camellia Sinensis Modulates Telomerase in Collagen-Induced
} Arthritis

\author{
Seyedeh Zohreh Jalali, ${ }^{1}$ and Farshid Saadat ${ }^{1,}$ \\ ${ }^{1}$ Faculty of Medicine, Guilan University of Medical Sciences, Rasht, Iran \\ "Corresponding author: Farshid Saadat, Pharm. D, PhD, Associate Professor of Medicinal Immunology, Department of Immunology, School of Medicine, Guilan University of \\ Medical Sciences, Rasht, Iran. E-mail: fsaadat@alumnus.tums.ac.ir \\ Received 2016 May 21; Revised 2016 August 12; Accepted 2017 February 08.
}

\begin{abstract}
Background: Rheumatoid arthritis is a chronic inflammatory disease with undefined pathogenesis. Some evidence suggests that telomerase is involved in the pathogenesis of this disease. This study was done to evaluate the therapeutic potency of Camellia sinensis stew (CSS) which rich in polyphenolic compounds with anti-inflammatory properties in experimental model of rheumatoid arthritis.

Methods: In this experimental study, collagen-induced arthritis (CIA) was induced by immunization of Lewis rats. The paws and knees were then removed for histopathology analysis. Moreover, Roentgenogram analysis of normal and arthritic hind paws was performed. Synovial cell harvested from biopsies of synovial tissue were used for measuring telomerase activity. The results were analyzed using SPSS version 16 software.

Results: Our data showed that CSS induced a significant reduction in paw edema. Histopathological findings showed reduced inflammatory responses and bone erosion in joints of treated rats. Moreover, CSS showed a significant reduction of telomerase activity compares with control group.

Conclusions: Our findings revealed that CSS should be considered as a therapeutic agent in experimental model of rheumatoid arthritis based on its telomerase activity suppression.
\end{abstract}

Keywords: Camellia Sinensis, Telomerase, Rheumatoid Arthritis

\section{Background}

Rheumatoid arthritis (RA) is an autoimmune disorder characterized by hyperplasia of synoviocytes leading to joint destruction and permanent deformity. While this disease has a worldwide distribution and increases in prevalence with age, its pathogenesis is not clearly understood [1]. Based on the resemblances between the joint pathology in CIA and RA, it suggests as a relevant murine model to search for new therapeutic agents against arthritis [2]. Although, nowadays therapeutic agents lessen the progression rate of the disease, joint damage typically occurs before patients are diagnosed. Although, the definite causes of RA are ambiguous, some evidence suggests that telomerase is involved in the pathogenesis of this disease [3, 4].

Telomerase is a ribonucleoprotein enzyme which enlarges the ends of chromosomes by adding repeated units of TTAGGG. An RNA component of this enzyme, called hTERT which serves as a template for addition of telomeric repeats. A number of reports have mentioned a relationship between degree of invasiveness and the increased telomerase activity. In an in vitro study, transfection of rheumatoid arthritis synovial fibroblasts with vectors expressing anti-sense oligonucleotide against the hTERT has led to cytolysis of these cells that exhibit high telomerase activity [5].

Green tea prepared from the dried leaves of Camellia sinensis, is drunk widely by people globally. There are many polyphenolic compounds in green tea among them, epigallocatechin gallate (EGCG) should be considered. These antioxidants polyphenols possess antiinflammatory properties. Fail to adequately control the underlying pathophysiology of RA suggesting that finding new therapeutic agents. In this study we evaluated the effects of Camellia sinensis dried leaves extract on telomerase activity in experimental collagen-induced arthritis.

\section{Methods}

In this experimental study, sixteen healthy adult male Lewis rats with average body weight (160 - $180 \mathrm{~g})$ were obtained from national institute of genetic engineering and biotechnology (NIGEB, Tehran, Iran). The rats housed in a controlled environment and determined food and water were divided at random into non-treated and treated groups with Camellia sinensis stew(CSS) $1 \mathrm{mg} / \mathrm{kg} / \mathrm{d}$. Animal care was in compliance with Iranian regulations on the 
protection of animals used for experimental and other scientific purposes and in accordance with the international principles for biomedical research involving animals, revised in 1985. Fine grade materials were obtained from Sigma-Aldrich (St. Louis, MO, USA).

For Induction of CIA, Bovine CII was emulsified with an equal volume of Freund's complete adjuvant (CFA). For induction CIA, on day one, $100 \mu \mathrm{L}$ of emulsion were injected intradermally at the base of the tail. A second injection of CII in CFA was administered on day 21. The orally administration of CSS were started on day 25 post-immunization and continued until final assessment on day 35 [6]. During this period, clinical examination was taken intermittently. The paws and knees were then removed for histopathological assay. Macroscopic scoring system using a scale from 0 to 4 for each paw was used to evaluate arthritis [7]. On day 35 , animals were anesthetized and killed. After fixation and decalcification of paws and knees, they stained with hematoxylin and eosin for histological examination. Joint damage was assessed as previously described [7]. According to the severity of damage, joint erosion was graded on a scale of 0 - 3 for each limb.

For radiography of normal and arthritic hind paws, the rats were anaesthetized with sodium pentobarbital ( 45 $\mathrm{mg} \mathrm{kg}^{-1}$, i.p.). Based on a score from 0 to 3 (score 0 , no bone damage; score 1, tissue swelling and edema; score 2, joint erosion; score 3, bone erosion and osteophyte formation) a blinded investigator performed radiograph score.

Biopsies of synovial tissue were obtained aseptically from the knee joints of rat. For harvesting the synovium from the knee joint, the patellar tendon with straight skin incision exposed. After cutting the patellar tendon transversely, and peeling the tendon, the infrapatellar fat pad from the femur and tibia was separated and then the synovium put into PBS in a Falcon tube. Finally, synovial tissue specimens were digested with $0.2 \%$ collagenase in highglucose DMEM plus 10\% FBS and antibiotics. Following overnight incubation at $37^{\circ} \mathrm{C}$, collected cells were plated and allowed to reach confluency at $37^{\circ} \mathrm{C}$ in a humidified atmosphere of $5 \% \mathrm{CO}_{2}$.

Telomerase activity of was measured by TRAPeze telomerase detection kit (Intergen, Inc., USA) according to the kit instructions. Briefly, using CHAPS lysis buffer, harvested cells from synovial tissue were lysed and for 30 minutes at $30^{\circ} \mathrm{C}$ the telomerase was first extended and then amplified by 30 cycles of PCR. By silver nitrate staining, the products of PCR were detected. Telomerase activity was calculated as the ratio of the intensity of telomerase ladders to the intensity of the 36-bp internal standard.

All data are expressed as the mean \pm SEM. The results of different experiment conditions were compared using independent sample t-test utilizing the SPSS (ver. 16) soft- ware, and the results were considered statistically significant at $\mathrm{P}<0.05$.

\section{Results}

The efficacy of RA therapy with CSS is presented in Figure 1. CIA developed in rat immunized with CII, and clinical signs (periarticular erythema and edema) of the disease first appeared in the hind paws between days 25 and 27 after CII challenge, with a 100\% incidence of CIA by day 27. The administration of CSS to arthritic rats could rapidly reverse paw edema. The difference between control group and treated rats on days $30-35$ of experiment was signifi$\operatorname{cant}(\mathrm{P}<0.000)$.

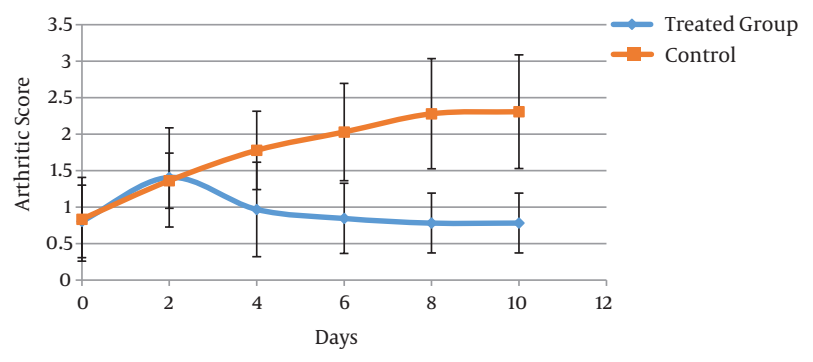

Figure 1. Arthritis Scores of Treated and Non-Treated (Control) Rats in Different Days After the Orally Administration of CSS on Day 25 Post-Immunization

Histological evaluation of the paws in the control animals reveals signs of severe arthritis along with inflammatory cells infiltrate (Figure 2). In treated group, histopathological assessment showed a reduced inflammatory cells infiltrate in the joints of treated rats, as well as markedly reduction in the number of osteoclasts, tissue edema and bone erosion indicated of its effectiveness for ameliorating synovial inflammation and prevention of joints destruction. The results of histopathology score in both treated and non-treated rats were shown in Table 1.

In addition, our radiographic finding didn't show any statistically differences between treated and non-treated arthritic hind paws $(P=0.05)$. The mean differences between treated and non-treated arthritic hind paws were $1.2812+0.80664$ vs. $2.25+0.99103$, respectively. The Xray results revealed that CSS-treated rats had more intact joint structure, as compared with their control counterparts (Figure 3 ).

Cells obtained from animal treated with orally administration of Camellia sinensis stew showed a significant reduction of telomerase activity (about 50\%) compared with control group; $1.0000 \mathrm{E} 2+0.95000$ vs. $52.5900+0.78000$, respectively $(\mathrm{P}<0.000)$ (Figure 4$)$. 


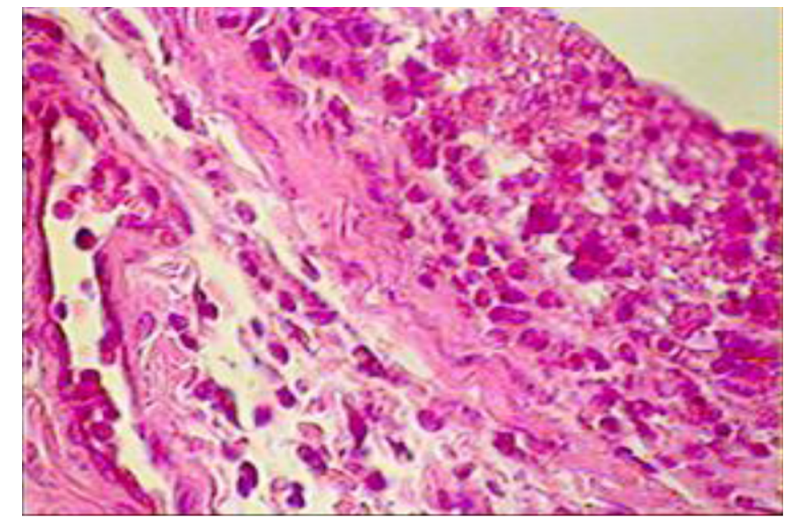

Control

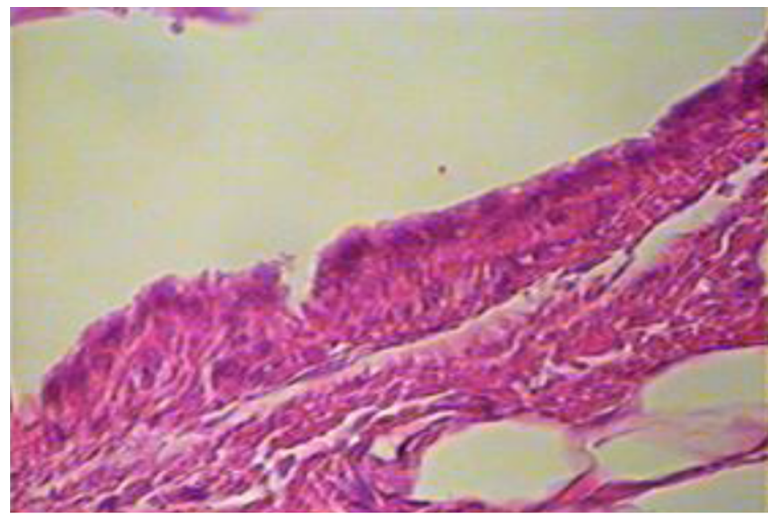

Treated Group

Figure 2. Hematoxylin and Eosin Staining of a Hind Joint of an Arthritic Animal Treated with CSS and Non-Treated Lewis Rat, a Control Rat with Collagen-Induced Arthritis, Depicted in $400 \times$ Magnifications

Table 1. Results of Arthritis, Histopathology and Radiology Scores As Well As Telomerase Activity in Treated and Control Rats

\begin{tabular}{|c|c|c|c|c|c|c|c|}
\hline \multirow[t]{2}{*}{ Variables } & \multirow[t]{2}{*}{ Group } & \multirow[t]{2}{*}{ Mean } & \multirow[t]{2}{*}{ Std. Deviation } & \multirow[t]{2}{*}{$\mathbf{t}$} & \multirow[t]{2}{*}{ Sig. (2-tailed) } & \multicolumn{2}{|c|}{ 95\% CI of the Difference } \\
\hline & & & & & & Lower & Upper \\
\hline \multirow{2}{*}{ Arthritis score } & Control & 2.4062 & 0.76692 & 5.284 & 0.000 & 0.96537 & 2.28463 \\
\hline & $\begin{array}{l}\text { Treated } \\
\text { group }\end{array}$ & 0.7812 & 0.41052 & & & & \\
\hline \multirow{2}{*}{ Histopathology score } & Control & 3.1250 & 0.83452 & 3.771 & 0.002 & 0.86256 & 3.13744 \\
\hline & $\begin{array}{l}\text { Treated } \\
\text { group }\end{array}$ & 1.1250 & 1.24642 & & & & \\
\hline \multirow{2}{*}{ Radiology score } & Control & 2.2500 & 0.99103 & \multirow{2}{*}{2.144} & \multirow{2}{*}{0.05} & \multirow{2}{*}{0.00021} & \multirow{2}{*}{1.93771} \\
\hline & $\begin{array}{l}\text { Treated } \\
\text { group }\end{array}$ & 1.2812 & 0.80664 & & & & \\
\hline \multirow{2}{*}{ Telomerase activity } & Control & $1.0000 \mathrm{E} 2$ & 0.95000 & 66.806 & 0.000 & 45.43964 & 49.38036 \\
\hline & $\begin{array}{l}\text { Treated } \\
\text { group }\end{array}$ & 52.5900 & 0.78000 & & & & \\
\hline
\end{tabular}

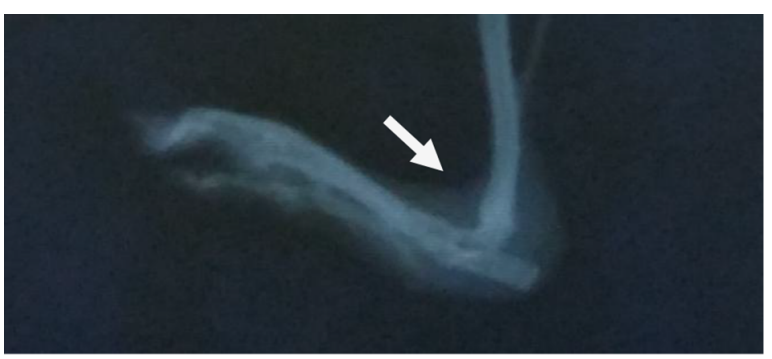

Control

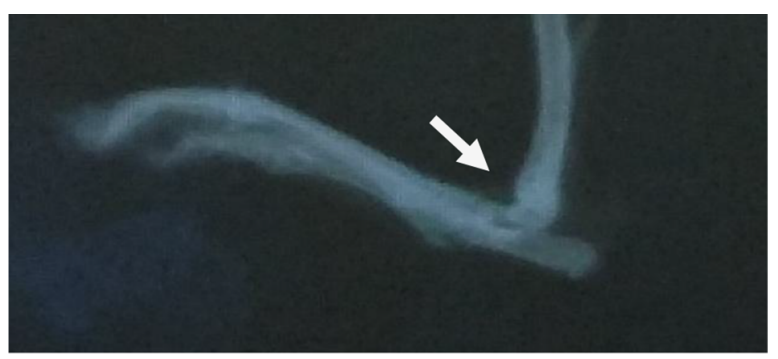

Treated

Figure 3. Representative Photographs of a Hind Joint of an Arthritic Animal Treated with CSS and Non-Treated Lewis Rat, a Control Rat with Collagen-Induced Arthritis. Arrows Indicate Differences in the Degree of Joint Damage Between Control and Treated Groups

\section{Discussion}

The recent investigations indicate that rheumatoid arthritis should not be considered as a benign disease. Zahedan J Res Med Sci. 2017; 19(2):e7210.
Moreover, it is associated with diminished long term survival. The clinical trials on different treatment strategies 
A

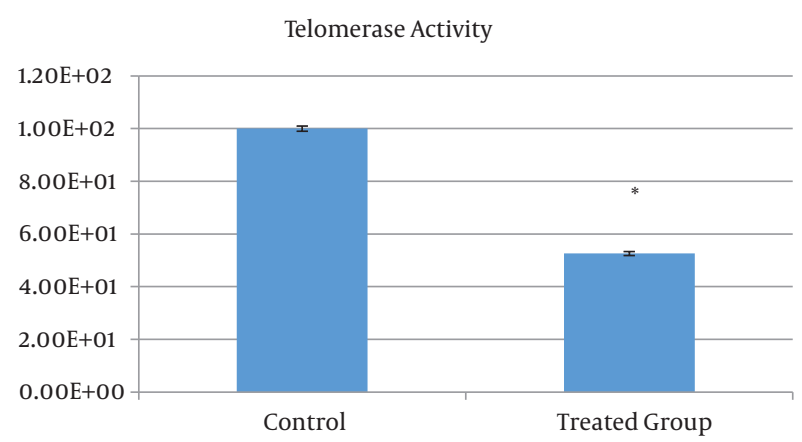

B

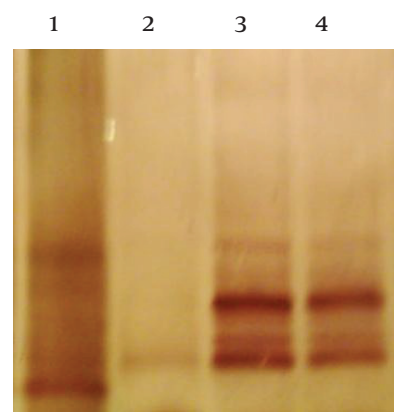

TRAP Product

Figure 4. Telomerase Activity of Synovial Cells of Treated and Control Rats (A).A Representative of Gel Electrophoresis for Telomerase Activity Measurement is Shown After the Telomerase in Cells Was Amplified by 30 Cycles of PCR and Stained by Silver Nitrate. Lane 1, Size Marker; Lane 2, Negative Control; Lane Treated Group; Lane 4 Control. (B)

in rheumatoid arthritis have shown that various types of medications are useful in the treatment of rheumatic diseases [8-10]. In this investigation, to imitate the clinical scenario of RA, CIA was induced in Lewis rats and subsequently, the anti-arthritic potency of Camellia sinensis stew was assessed in mentioned experimental model. This beverage is being used for many years and possesses antioxidant activities, anti-inflammatory, anti-apoptotic properties and protective effects in autoimmune animal models [11-15].

Here, we demonstrate that treatment with CSS reduces the development of clinical signs and the degree of joint injury in CIA model. Moreover, reduced inflammatory cells infiltrate in the joints of treated animals with type II collagen was occurred due to down-regulation of proinflammatory cytokines and chemokine receptor in synovial tissues [16]. All of these findings support the view that CSS attenuates the degree of arthritis and joint damage caused by collagen in this model.
In RA, osteoclasts abnormally activated due to interaction with synovial fibroblasts and helper $\mathrm{T}$ cells and, in turn induce bone destruction. In our findings, a reduction in the clinical scores of joint inflammation and joint erosion were in agreement with the result of trial in which the green tea polyphenol epigallocatechin-3-gallateas a potent antioxidant improves autoimmune arthritis by both regulation of TH17 regulatory cells and inhibition of osteoclasts development [17]. Moreover, there is more evidence that polyphenols in green tea, particularly EGCG, inhibit matrix metalloproteinase activity which plays a fundamental role in pathogenesis of many diseases such as RA [18]. Although, joint erosion in CSS-fed animals reduced, the level of significance in the radiological index of the joints in treated rats compared to control could not be achieved. It might be attributed to numerous factors including the number of animals in each group and the delay in treatment schedule.

To investigate the anti-telomerase effect of CSS, we used the synovial fibroblasts. Synovial cells proliferate and transform the normally synovium into an invasive pannus. This architecture creates a hypoxic microenvironment and, in turn, through the increased production of reactive oxygen species induce inflammation [19]. Since, these cells contribute to chronic inflammatory responses in RA. Data obtained from telomerase assay exhibited that CSS induced reduction of telomerase activity compares with control group. Since the regulatory role of telomerase on cell proliferation has been recognized, its inhibition by various molecules has been considered by many investigators as a new approach for cancer therapy. Moreover, the crucial role of telomerase activity in other feature of neoplastic phenotype like invasion, apoptosis resistancy and so on were determined in some cancer models. Based on the presence of two E-box boxes on the hTERT gene promoter which are the binding sites of transcription factors like c-Myc, the activation of telomerase was controlled [20, 21]. Wang et al. [22] found that c-Myc protein level was significantly decreased after EGCG treatment, which was accompanied by the reduction of the telomerase activity. However, molecular mechanisms by which CSS induces reduction of telomerase activity are thus still called for.

In conclusion, we show that CSS effectively suppresses collagen arthritis and, a potent inhibitory effect on telomerase activity and based on its high tolerability, it can be recommended for long-term administration, in the future investigations.

\section{Footnotes}

Authors' Contribution: All authors had equal role in design, work, statistical analysis and manuscript writing. 
Conflict of Interest: The Authors declare that they have no conflict of interests.

\section{References}

1. Gibofsky A. Overview of epidemiology, pathophysiology, and diagnosis of rheumatoid arthritis. Am J Manag Care. 2012;18(13 Suppl):S295302. [PubMed: 23327517].

2. Larsson P, Kleinau S, Holmdahl R, Klareskog L. Homologous type II collagen-induced arthritis in rats. Characterization of the disease and demonstration of clinically distinct forms of arthritis in two strains of rats after immunization with the same collagen preparation. Arthritis Rheum. 1990;33(5):693-701. doi: 10.1002/art.1780330512. [PubMed: 2346524].

3. Natarajan V, Madhan B, Tiku ML. Intra-Articular Injections of Polyphenols Protect Articular Cartilage from Inflammation-Induced Degradation: Suggesting a Potential Role in Cartilage Therapeutics. PLoS One. 2015;10(6):e0127165. doi: 10.1371/journal.pone.0127165. [PubMed: 26046639].

4. Yamanishi Y, Hiyama K, Ishioka S, Maeda H, Yamanaka T, Kurose Y, et al. Telomerase activity in the synovial tissues of chronic inflammatory and non-inflammatory rheumatic diseases. Int J Mol Med. 1999;4(5):513-7. doi: 10.3892/ijmm.4.5.513. [PubMed: 10534574].

5. Chen SY, Shiau AL, Shieh GS, Su CH, Lee CH, Lee HL, et al. Amelioration of experimental arthritis by a telomerase-dependent conditionally replicating adenovirus that targets synovial fibroblasts. Arthritis Rheum. 2009;60(11):3290-302. doi: 10.1002/art.24940. [PubMed: 19877026].

6. Cuzzocrea S, Mazzon E, Di Paola R, Muia C, Crisafulli C, Dugo L, et al. Glycogen synthase kinase-3beta inhibition attenuates the degree of arthritis caused by type II collagen in the mouse. Clin Immunol. 2006;120(1):57-67. doi: 10.1016/j.clim.2006.03.005. [PubMed: 16631408].

7. Mirshafiey A, Saadat F, Attar M, Di Paola R, Sedaghat R, Cuzzocrea S. Design of a new line in treatment of experimental rheumatoid arthritis by artesunate. Immunopharmacol Immunotoxicol. 2006;28(3):397-410. doi: 10.1080/08923970600927447. [PubMed: 16997789].

8. Charles-Schoeman C, Gonzalez-Gay MA, Kaplan I, Boy M, Geier J, Luo Z, et al. Effects of tofacitinib and other DMARDs on lipid profiles in rheumatoid arthritis: implications for the rheumatologist. Semin Arthritis Rheum. 2016;46(1):71-80. doi: 10.1016/j.semarthrit.2016.03.004. [PubMed: 27079757].

9. Scott IC, Ibrahim F, Simpson G, Kowalczyk A, White-Alao B, Hassell A, et al. A randomised trial evaluating anakinra in early active rheumatoid arthritis. Clin Exp Rheumatol. 2016;34(1):88-93. [PubMed: 26842950].

10. Genovese MC, van Vollenhoven RF, Pacheco-Tena C, Zhang Y, Kinnman N. VX-509 (Decernotinib), an Oral Selective JAK-3 Inhibitor, in Combination With Methotrexate in Patients With Rheumatoid Arthritis. Arthritis Rheumatol. 2016;68(1):46-55. doi:10.1002/art.39473. [PubMed: 26473751].
11. Lorenz M. Cellular targets for the beneficial actions of tea polyphenols. Am J Clin Nutr. 2013;98(6 Suppl):1642S-50S. doi: 10.3945/ajcn.113.058230. [PubMed: 24172299].

12. Lambert JD, Elias RJ. The antioxidant and pro-oxidant activities of green tea polyphenols: a role in cancer prevention. Arch Biochem Biophys. 2010;501(1):65-72. doi: 10.1016/j.abb.2010.06.013. [PubMed: 20558130].

13. Hong J, Smith TJ, Ho CT, August DA, Yang CS. Effects of purified green and black tea polyphenols on cyclooxygenase- and lipoxygenasedependent metabolism of arachidonic acid in human colon mucosa and colon tumor tissues. Biochem Pharmacol. 2001;62(9):1175-83. doi: 10.1016/S0006-2952(01)00767-5. [PubMed: 11705450].

14. Hsu SD, Dickinson DP, Qin H, Borke J, Ogbureke KU, Winger JN, et al. Green tea polyphenols reduce autoimmune symptoms in a murine model for human Sjogren's syndrome and protect human salivary acinar cells from TNF-alpha-induced cytotoxicity. Autoimmunity. 2007;40(2):138-47. doi: 10.1080/08916930601167343. [PubMed: 17364504].

15. Xiao J, Ho CT, Liong EC, Nanji AA, Leung TM, Lau TY, et al. Epigallocatechin gallate attenuates fibrosis, oxidative stress, and inflammation in non-alcoholic fatty liver disease rat model through TGF/SMAD, PI3 K/Akt/FoxO1, and NF-kappa B pathways. Eur J Nutr. 2014;53(1):187-99. doi: 10.1007/s00394-013-0516-8. [PubMed: 23515587].

16. Ramadan G, El-Beih NM, Talaat RM, Abd El-Ghffar EA. Antiinflammatory activity of green versus black tea aqueous extract in a rat model of human rheumatoid arthritis. Int J Rheum Dis. 2015 doi: 10.1111/1756-185X.12666. [PubMed: 25964045].

17. Lee SY, Jung YO, Ryu JG, Oh HJ, Son HJ, Lee SH, et al. Epigallocatechin3-gallate ameliorates autoimmune arthritis by reciprocal regulation of T helper-17 regulatory $\mathrm{T}$ cells and inhibition of osteoclastogenesis by inhibiting STAT3 signaling. J Leukoc Biol. 2016;100(3):559-68. doi: 10.1189/jlb.3A0514-261RR. [PubMed: 26957211].

18. Oka Y, Iwai S, Amano H, Irie Y, Yatomi K, Ryu K, et al. Tea polyphenols inhibit rat osteoclast formation and differentiation.J Pharmacol Sci. 2012;118(1):55-64. doi: 10.1254/jphs.11082FP. [PubMed: 22186621].

19. Fearon U, Canavan M, Biniecka M, Veale DJ. Hypoxia, mitochondrial dysfunction and synovial invasiveness in rheumatoid arthritis. Nat Rev Rheumatol. 2016;12(7):385-97. doi: 10.1038/nrrheum.2016.69. [PubMed: 27225300].

20. Kyo S, Takakura M, Taira T, Kanaya T, Itoh H, Yutsudo M, et al. Sp1 cooperates with c-Myc to activate transcription of the human telomerase reverse transcriptase gene (hTERT). Nucleic Acids Res. 2000;28(3):66977. doi: 10.1093/nar/28.3.669. [PubMed: 10637317].

21. Oh S, Song YH, Yim J, Kim TK. Identification of Mad as a repressor of the human telomerase (hTERT) gene. Oncogene. 2000;19(11):1485-90. doi: 10.1038/sj.onc.1203439. [PubMed: 10723141].

22. Wang M, Lei YX. Effects of tea polyphenols on proliferation and apoptosis of cadmium-transformed cells. IntJClin Exp Med. 2015;8(2):305462. [PubMed: 25932282]. 